\title{
Structural Fatigue Life Prediction Based on ANSYS Random Vibration Analysis
}

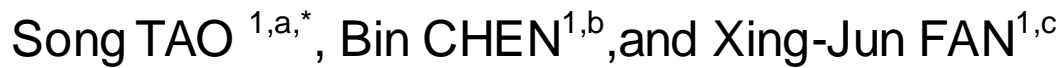 \\ ${ }^{1}$ College of Basic Education, National University of Defense Technology, Changsha, China \\ a taomugong@126.com, bchenbin_gfkd@sina.com, ${ }^{\mathrm{c}} 724072166 @ q q . c o m$ \\ ${ }^{*}$ Corresponding author
}

Key words: Life prediction, ANSYS, Random vibration, Linear cumulative damage theory, Three interval method

\begin{abstract}
Fatigue failure is one of the main reasons for the engineering structure's failure, and it is of great significance to predict fatigue life of structure. This paper briefly introduced the principle of stochastic fatigue failure calculation analysis, and analyzed random vibration of a T-shaped structure by using ANSYS software. Under a given load combined with linear cumulative damage theory and three interval method proposed by Steinberg, the fatigue life of the structure is predicted.
\end{abstract}

\section{Introduction}

It is important to prevent the fatigue failure of the material and ensure the safety and stability of engineering structure during the design life. Since the first half of nineteenth Century, people have studied the fatigue of materials and structures for more than 160 years [1,2],but in our life, there are still some catastrophic accidents caused by fatigue failure of structural members. The study shows that fatigue failure is one of the main causes of huge economic losses[3].Therefore, it is of great significance to study the fatigue of the material and structural members and predict the fatigue life of the structure.

ANSYS is one of the most widely used finite element analysis softwares at home and abroad, and its powerful function and good user interface are widely welcomed by the mas ses. ANSYS software is very good to achieve pre-processing and post-processing and is widely used in aerospace, machinery manufacturing, civil engineering and other fields. In this paper, ANSYS is used to analyze the random vibration of an engineering structure. Based on the results of the post-processing, the fatigue failure of the structure is calculated, and the fatigue life prediction of the structure is realized.

\section{Random Fatigue Failure Analysis Principle}

The cumulative damage failure is one of structure's failure modes in the random working state, which is the cumulative damage to the micro damage, the component fatigue failure occurs until the cumulative damage to a value. For deterministic vibration and constant stress amplitude, the fatigue failure is calculated according to the $\mathrm{S}-\mathrm{N}$ curve of material to determine. For random vibration, each cycle will cause cumulative damage, which is the same with the deterministic vibration fatigue analysis theory. In the random vibration environment, fatigue calculation is relatively complex because that the response of the structure is a random quantity. In the engineering, the three interval method proposed by Steinberg is widely used, that is, the structure of the Von Mises stress response obeys Gauss distribution to simplify the calculation, expressed as:

The stress value between $-1 \sigma$ and $1 \sigma$ takes up $68.3 \%$ of the total time;

The stress value between $-2 \sigma$ and $2 \sigma$ takes up $68.3 \%$ of the total time;

The stress value between $-3 \sigma$ and $3 \sigma$ takes up $68.3 \%$ of the total time;

The stress value more than $3 \sigma$ occurs within $0.27 \%$ of the time, so we assume that it does not cause any damage.

Three interval method based on fixed amplitude, it is assumed that it experiences $\mathrm{n}$ cycles so 
$\mathrm{n} / N$ part of structural fatigue life is consumed. According to the linear cumulative damage theory, the damage caused by $n_{i}$ cycles under the condition of the load $\sigma_{i}$ is expressed as

$$
D=\sum_{i=1}^{n} \frac{n_{i}}{N_{i}}
$$

Where $N_{i}$ is the number of fatigue cycles corresponding to the current load level $\sigma_{i}$.

Research has shown that the critical damage value of testspecimens is about 1 for random $\operatorname{load}^{[2]}$,so it can be considered that when fatigue failure of structure happens,

$$
D=1
$$

In random vibration environment, the Von Mises stress responses are divided into three stress level, $1 \sigma, 2 \sigma, 3 \sigma$.According to the available fatigue curve, structural material stress level $1 \sigma, 2 \sigma, 3 \sigma$ respectively correspond to number of permits circulation $N_{1 \sigma} 、 N_{2 \sigma} 、 N_{3 \sigma}$.In the random vibration structure fatigue time $T$, the actual number of cycles is expressed as

$$
\begin{gathered}
\mathrm{n}_{1 \sigma}=0.683 v^{+} T \\
\mathrm{n}_{2 \sigma}=0.271 v^{+} T \\
\mathrm{n}_{3 \sigma}=0.0433 v^{+} T
\end{gathered}
$$

Where $\mathrm{n}_{1 \sigma}, \mathrm{n}_{2 \sigma}, \mathrm{n}_{3 \sigma}$ represent the number of cycles under the stress level $1 \sigma, 2 \sigma, 3 \sigma$ respectively; $v^{+}$is statistical average frequency of Von Mises stress, which is defined as [4]

$$
v^{+}=\frac{\mathrm{f}_{\max }-\mathrm{f}_{\min }}{2}
$$

Using (3) into (1), the formula for calculating the overall damage of the structure is expressed by

$$
D=\frac{0.683 v^{+} T}{N_{1 \sigma}}+\frac{0.271 v^{+} T}{N_{2 \sigma}}+\frac{0.0433 v^{+} T}{N_{3 \sigma}}
$$

Using (5) into (2), the fatigue life of the structure can be obtained as follows:

$$
T=1 /\left(\frac{0.683 v^{+}}{N_{1 \sigma}}+\frac{0.271 v^{+}}{N_{2 \sigma}}+\frac{0.0433 v^{+}}{N_{3 \sigma}}\right)
$$

The above calculation is for the structure of a single material composition. When the structure is composed of a variety of materials, the shortest material fatigue failure time is the life of the structure.

\section{Analysis \& Calculation of ANS YS}

\subsection{ANS YS Modeling and Finite Element Analysis}

A T-shaped fixture is made up of aluminum alloy plate, and the geometric model diagram is shown in Fig.1.The bottom of the fixture is fixed, which is subjected to random excitation in the horizontal direction. The random vibration load is the acceleration spectrum, the load curve is shown in Fig.2. 


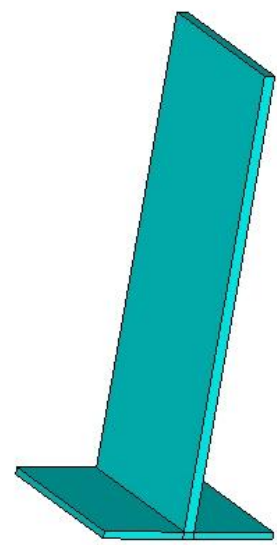

Fig.1 T-shaped fixture

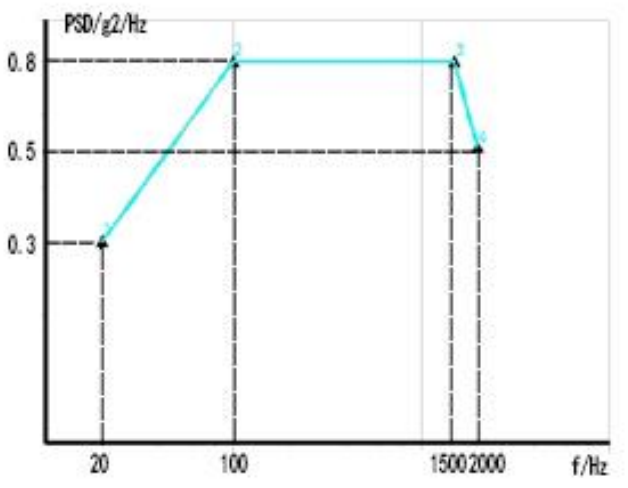

Fig.2 load curve

According to the reference [5], the mechanical properties of the aluminum alloy are shown in Table 1.In the finite element software ANSYS, the modal analysis of the structure is carried out, and the natural frequency within $2000 \mathrm{~Hz}$ of the structure is obtained, which is shown in Table 2.According to the results of modal analysis and the random vibration analysis, the $1 \sigma$ displacement solution of the structure is obtained. The $1 \sigma$ stress distribution is shown in Fig.3.

Table 1 Mechanical properties of aluminum alloy

\begin{tabular}{|c|c|c|c|}
\hline Material & Elastic modulus $(G P a)$ & Poisson ratio & Density $\left(\mathrm{kg} \cdot \mathrm{m}^{-3}\right)$ \\
\hline Aluminum alloy & 69 & 0.33 & 2700 \\
\hline
\end{tabular}

Table 2 Natural frequency of the model

\begin{tabular}{lllllll}
\hline Order number & 1 & 2 & 3 & 4 & 5 & 6 \\
\hline Natural frequency & 79.259 & 404.91 & 494.04 & 1153.5 & 1288.7 & 1381.8 \\
\hline
\end{tabular}

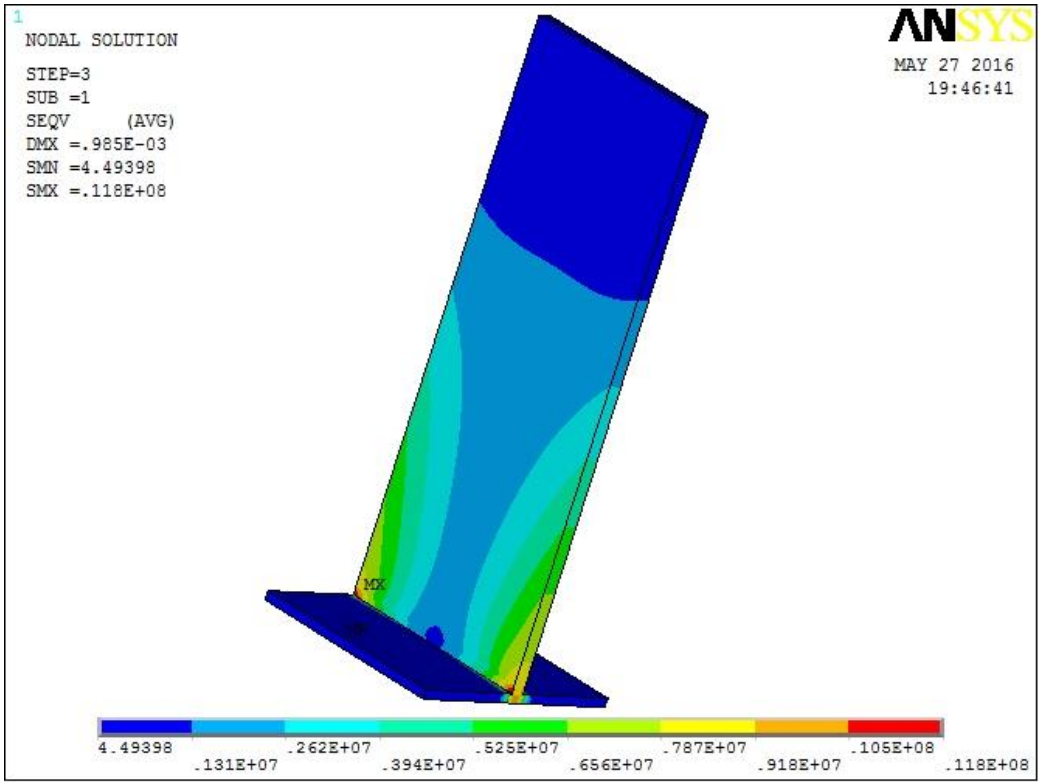

Fig. $31 \sigma$ stress distribution 


\subsection{Failure Calculation}

According to the results of ANSYS random vibration analysis, it is known that the maximum stress in the structure occurs at boundary of the junction of the horizontal plate and the vertical plate, and the stress value is $0.118 e 8 \mathrm{~Pa}$.According to the reference [4], the $\mathrm{S}-\mathrm{N}$ relationship of aluminum plate between $10^{3}$ and $10^{7}$ is expressed as

$$
\log N=57.7876-6.6489 \log \sigma
$$

$v^{+}=990 \mathrm{~Hz}$, which is obtained by formula (4) and the applied acceleration spectrum, combined with formula (6)and formula (7), fatigue life of the structure is shown in table 3.

Table 3 Fatigue life of the T-shaped fixture

\begin{tabular}{lllll}
\hline structure & $N_{1 \sigma}$ & $N_{2 \sigma}$ & $N_{3 \sigma}$ & Life $T(s)$ \\
\hline T-shaped fixture & $5.8528 \mathrm{e} 10$ & $5.8324 \mathrm{e} 8$ & $3.9358 \mathrm{e} 7$ & $6.4073 \mathrm{e} 5$ \\
\hline
\end{tabular}

Therefore, fatigue life of the T-shaped fixture in a given random vibration environment is estimated to be $6.4073 \mathrm{e} 5 \mathrm{~s}$.

\section{Conclusions}

This paper has analyzed random vibration of the T-shaped fixture by using the finite element software ANSYS, obtained the random response of the structure and the position of maximum stress according to the stress nephogram. Based on the results of random vibration analysis, the fatigue life of the structure is predicted by using the linear cumulative damage theory and three section method proposed by Steinberg, which provides a reference for structure application in engineering practice.

\section{References}

[1] Zheng.X.L., Material fatigue theory and engineering application, Beijing, Science Press, 2013.

[2] Yin. Z.P., Structural fatigue and fracture, Xi'an, Northwestern polytechnical university press, 2012.

[3] Fort, N.E., et al. Metal Fatigue, J. Journal of Engineering Materials \& Technology. 42.1(1975): 96.

[4] Chen. X.Q., Li. C., Life prediction of structures under random vibration environment, J. Modern machinery.1(2007):15-17

[5] Wang. S.C., Deng. Z.X., Shen.W.D.et al, Analysis on intrinsic properties of orthotropic honeycomb sandwich plates with four edges simply supported, J. Journal of Vibration and shock.31.9(2012):73-77. 